\title{
Recent progress in the field of optical coatings for Fabry-Perot interferometers and filters
}

\section{E. Pelletier}

\section{Laboratoire d'Optique des Surfaces et des Couches Minces} E.N.S. de Physique de Marseille - Domaine Universitaire de St Jérôme 13397 Marseille Cedex 20. France

\begin{abstract}
During the last years, considerable improvements occurred in the field of multidielectric spectral filters. Nowadays we can conceive and realize practically any filtering system meeting extremely precise requirements necessary for optical instruments and particularly for astronomy. We will give here a short overview of the multiple factors leading to these improvements.
\end{abstract}

\section{Deposition techniques}

Now we know how to realize layers made of dielectric materials such as metallic oxides with excellent optical and mechanical properties. In the optical industry, the more generally used technique is vacuum reactive evaporation with the main inconvenient of leading to relatively low compacted layers absorbing the ambient moisture when in contact with air. lintroducing an ion gun in the vacuum chamber to bombard the growing layer we can make the coatings more compact and quite completely eliminate the shift due to moisture adsorption. This deposition technique is known as Ion Assisted Deposition (IAD). A more sophisticated technique consists in using a second ion gun to obtain a sputtering (and not an evaporation as above) of the coating material: Dual Ion Beam Sputtering (DIBS). The industrial development of these techniques is essentially slowed down by the problem of manufacturing costs.

Eliminating the shift of the optical properties between vacuum and air makes the manufacturing of the multilayers simpler and the performances of the filters obtained in this way remain stable versus time. For example, the centering of narrow band filters can be guaranteed for many years.

\section{Synthesis}

An optical instrument, for astronomy for example, involves manufacturing of filters with spectral characteristics strictly determined on an eventually very large spectral range. This can be obtained only if, prior to fabrication, we are able to calculate the structure (i.e. the formula: indices and thicknesses of each layer) of the stack which will give rise to the best adapted interference phenomena. In this calculation, we must obviously take into account the manufacturing requirements. In particular, a high accuracy knowledge of the complex refractive 
indices of the layers of the various materials that we are able to deposit by IAD or by DIBS with a good reproducibility is mandatory.

When the problems of the exact knowledge of the optical constants are solved, it remains to solve the calculation problems. If in industry, except for some particular cases, most of the optical coatings do not include more than about ten layers, for special applications we have frequently to do the synthesis of more complex coatings, involving some $\mathbf{4 0}$ or even $\mathbf{8 0}$ layers. Knowing that the search for solutions can be made only by techniques of successive approximations, we can conceive how it is interesting to develop powerful algorithms on modern fast computers. To make their fabrication easier, the coatings are made of a minimum number of materials (very often two materials of indices as different as possible) and the aim of the computing program is to adjust the number of the layers and their thickness to meet the required optical properties.

\section{Optical monitoring}

The realization of optical multilayer coatings often gives rise to difficult problems. We must control the refractive index and the thickness of the layers during their deposition. For instance with a coating intended for the visible spectrum, the layer thicknesses are of about 20 to $500 \mathrm{~nm}$ and we must respect the values determined by the synthesis with an accuracy better than one nanometer, even if the stack is made of $\mathbf{5 0}$ of more layers. For this accuracy, only optical methods can be used to control the formation of the stack. It is often better to use a direct monitoring on the very substrate to coat rather than on a test sample. With the modern methods of spectrophotometry we can easily have a record of the spectral profile (that is in general the transmittance for normal incidence) of the growing stack each 0.1 second. Moreover we must stop the deposition of a layer at the precise moment when the required thickness is attained. This implies the choice of a stop criterion. For this, we can theoretically use the coincidence between the expected and the measured profiles. This method gives excellent results but is of difficult use. Indeed, we must simultaneously meet several conditions namely: the measured spectral profiles must be free of experimental errors and the calculated profiles, for each of the successive layers, are well defined objectives providing an accessible goal.

That is to say that the simulation calculation must account for the effective optical properties of the layers under vacuum and that, for the monitoring of the layer number i, we know, with a good precision, the parameters (indices and thicknesses) of the (i-1) layers constituting the stack already realized. The success of the manufacturing thus obviously implies a perfect mastering of the technology but also of the physics of thin layers.

For all these reasons, we often prefer an optical monitoring method of more simple use. Instead of exploiting the absolute transmission measurements on a wide spectral range, we can indeed be satisfied only by using a relative measurement, for a single wavelength previously chosen. So we find here the method well known as "turning value monitoring" which uses as stop criteria the extrema occurring with time. The better precision is obtained with the techniques of derivation versus time or versus wavelength. 


\section{Production tolerances}

When a complex layer stack is conceived by synthesis, what about the realization tolerances of such a stack? Is it really possible to manufacture a 50-layer coating with a precision of about $0.1 \mathrm{~nm}$ on the thickness of each layer? In general the answer is negative, but fortunately the question of tolerances is not well addressed. When approaching this problem, we must consider simultaneously the stack to be realized and the detail of the monitoring program that will be used. Instead of considering the tolerances of realization on the thicknesses, we must determine the tolerances on the respect of the stop criterion of the layers, knowing the sensitivity of the apparatus. It is essential to understand that, with a direct monitoring, as the multilayer deposition proceeds, the errors disturb more or less the monitoring of the following layers. In some cases, the effect is positive since all is going as if the light passing through the stack could detect the errors and adjust accordingly the stop criterion for the thickness of the layer under control in such a way as to induce the best adapted interference state. There is then an automatic error compensation and the stack is much more easy to produce as that could be thought when neglecting the role of cumulative effects - In this way we introduce the concept of a stable monitoring associated with a stack and well defined optical properties. Let us specify that each coating is intended to meet particular specifications in one or several well determined spectral ranges and that it is interesting to carry out the optical monitoring precisely in these useful spectral ranges.

A great improvement for the synthesis of a filter consists in selecting among the possible solutions the ones that do not exhibit insolvable realization problems. A simple and classical example gives a good illustration of this remark: it concerns the narrow band interference filters made out of quarterwave stacks. The useful optical properties are centered on lo; the monitoring uses this same wavelength lo and it can act as a real time computer that rectifies the errors as they occur.

\section{Minimization of losses}

In the preceding sections we have essentially dwelled on the recent advances in the problems of synthesis and manufacturing of layer stacks. However we cannot forget the improvements concerning the minimization of losses in the multilayers. These losses are due to scattering and to absorption of light. Scattering originates from roughnesses at the interfaces and from index heterogeneities in the bulk of the layers. Absorption is essentially due to impurities in the materials constituting the layers. Numerous works deal with all these phenomena. Several laboratories in the world developed important and powerful instruments allowing an accurate determination of losses even when as low as $10^{-6}$ of the incident flux.

These whole phenomena are now well understood and mastered, allowing the manufacturing of coatings with optimum performances when necessary. Unfortunately the costs of fabrication often restrict the capabilities that could be offered to the users of such coatings. 